\title{
Gradhiva
}

GRADHIV

Revue d'anthropologie et d'histoire des arts

28 | 2018

Capsules temporelles

\section{Temporalités encapsulées dans les peintures aborigènes du musée du quai Branly}

Encapsulated temporalities in the aboriginal paintings of the musée du quai Branly

\section{Arnaud Morvan}

\section{OpenEdition}

Journals

Édition électronique

URL : http://journals.openedition.org/gradhiva/3685

DOI : 10.4000/gradhiva.3685

ISSN : $1760-849 x$

Éditeur

Musée du quai Branly Jacques Chirac

Édition imprimée

Date de publication : 28 novembre 2018

Pagination : $50-75$

ISBN : 978-2-35744-111-8

ISSN : 0764-8928

\section{Référence électronique}

Arnaud Morvan, «Temporalités encapsulées dans les peintures aborigènes du musée du quai

Branly », Gradhiva [En ligne], 28 | 2018, mis en ligne le 28 novembre 2020, consulté le 06 janvier 2021.

URL : http://journals.openedition.org/gradhiva/3685; DOI : https://doi.org/10.4000/gradhiva.3685

(c) musée du quai Branly 
Temporalités encapsulées dans les peintures aborigènes du musée du quai Branly

par Arnaud Morvan

À partir d'une réflexion sur l'incorporation de l'histoire coloniale dans les œuvres d'art et les objets rituels aborigènes, l'article examine l'intégration d'une peinture contemporaine de l'artiste kija Paddy Nyunkuny Bedford à l'architecture du musée du quai Branly. Il s'agit de déterminer si la temporalité non-linéaire aborigène qui met plusieurs temps en présence relève d'une forme de capsule temporelle, ou bien si celle-ci n'est qu'une projection européenne reliant des pratiques non-occidentales à un passé mythique. L'œuvre de Bedford semble juxtaposer différents modes d'inscription de l'événement dans le paysage, l'un mythique, l'autre historique, et matérialiser le passage entre les dimensions virtuelles et actuelles de la mémoire.

mots clés

art, aborigène, musée,

capsules temporelles,

histoire coloniale 
1. "Sur eux, est gravée ou dessinée la marque totémique. C'est donc cette marque et elle seule qui leur confère le caractère sacré. " (Durkheim 2008 [1912] : 172)

2. "Leur caractère sacré tient à la fonction de signification diachronique qu'ils sont seuls à assurer dans un système qui, parce que classificatoire, est complètement étalé dans une synchronie qui réussit même à s'assimiler la durée. " (Lévi-Strauss 2008: 819)
Dans Les Formes élémentaires de la vie religieuse publié en 1912 , Émile Durkheim utilise les matériaux ethnographiques arrernte (aranda ou arunta) collectés par Baldwin Spencer et Francis Gillen en Australie centrale pour fonder l'une des toutes premières anthropologies du temps, basée sur l'étude du système totémique australien. L'ouvrage, qui affirme la primauté du social sur le religieux et l'existence du temps social comme catégorie de l'entendement, place au centre de sa démonstration une analyse des objets sacrés et secrets aborigènes, les churinga, comme prototypes de l'emblème totémique et d'une dimension sacrée de la société. Le terme churinga ou tjuringa désigne la manifestation tangible d'un être totémique, sous forme de pierre ou de tablettes gravées ou lisses, utilisées pendant les cérémonies religieuses des groupes arrernte du centre de l'Australie. Ces objets gardés dans des grottes ou d'autres lieux secrets sont dits contenir les stocks d'âmes ou «esprits-enfants ", déposés par les êtres mythiques, et s'incarner dans les futurs enfants à naître lorsque des femmes passent à leur proximité. La révélation d'un churinga, qui se produit graduellement durant le parcours initiatique, permet d'entrer en contact avec l'Alcheringa, une temporalité mythique lors de laquelle les êtres totémiques ont créé le monde et les différentes catégories d'existants. Au cours d'un rituel complexe, l'initié est ainsi mis en présence de son origine totémique (Moisseeff 1995; Stanton 2001; Mary 2012). Or une telle opération temporelle est difficilement compréhensible si on se réfère à la théorie durkhemienne du churinga comme symbole, c'est-à-dire comme emblème du clan manifesté au cours d'un sacrifice ${ }^{\mathbf{1}}$. Claude Lévi-Strauss, revenant sur les churinga dans le chapitre de La Pensée sauvage intitulé "Le temps retrouvé», y voit plutôt un opérateur permettant de connecter des temporalités différentes pour intégrer de nouveaux événements, à la manière d'une archive dans les cultures occidentales ${ }^{2}$.

Les peintures rupestres dont les motifs sont reproduits sur le sable et les churinga cachés dans les grottes semblent former des capsules temporelles analogues à celles qui furent inventées aux États-Unis par des ingénieurs puis des artistes (Jarvis 2003, Di Liberti 2013). L'espace circonscrit de la cache renfermant les churinga d'un clan n'est pas sans rappeler l'espace-temps clos d'une boîte renfermant des fragments matériels d'un temps révolu, destinés aux générations futures, et le churinga lui-même ressemble à un de ces objets gravés que les concepteurs de capsules temporelles enfouissent sous terre ou envoient dans l'espace. Peut-on expliquer ainsi la fascination qu'ils exercent chez les amateurs d'«arts premiers »? Plus de cent ans après la publication de Durkheim et l'âge d'or des débats anthropologiques sur le totémisme, l'art aborigène contemporain soulève toujours un vif intérêt: issu de la plus ancienne tradition artistique du monde (certaines pratiques montrent une continuité avec des techniques vieilles de 40000 ans), il en est aussi, depuis son entrée sur le marché de l'art dans les années 1980, l'une des expressions les plus actuelles (Myers 2002; McLean [éd.] 2011, Morvan 2013). Mais il fait souvent l'objet de jugements «allochroniques» qui le rattachent à une temporalité culturellement séparée de la nôtre (Fabian 1983; Kaehr et Hainard 1985; Gell 1992). La notion de capsule temporelle permet-elle cependant de décrire la pluralité des temporalités qui se connectent dans une œuvre aborigène et leur capacité à intégrer la discontinuité de l'événement? La temporalité non-linéaire aborigène qui met plusieurs temps en présence relève-t-elle d'une forme de capsule temporelle, ou celle-ci n'est-elle pas 
une projection européenne sur les pratiques aborigènes pour les ramener dans un passé mythique? Si, parmi les œuvres aborigènes, les musées et les galeries ont privilégié les churinga et les peintures sur toile, n'est-ce pas parce qu'ils exprimaient une temporalité hétérogène sur fond de rupture catastrophique, celle d'un monde risquant de disparaître?

Je soulèverai ces questions en examinant la commande publique d'art aborigène faite par le musée du quai Branly au début des années 2000 à huit artistes aborigènes. Je me concentrerai plus particulièrement sur l'œuvre de Paddy Bedford, artiste kija de la région du Kimberley oriental, dans le nordouest de l'Australie. L'œuvre de cet artiste, reconnu internationalement de son vivant, a pour particularité d'intégrer des références à la fois au temps mythique de la création et à des événements de l'histoire coloniale de la région. En retraçant la trajectoire de la création de Bedford pour le musée et en examinant ses origines rituelles et ses fondements conceptuels, je décrirai les dynamiques temporelles qui la constituent.

\section{La commande publique d'art aborigène du musée du quai Branly}

Le musée du quai Branly naît en 1996 en rassemblant les collections extra-européennes nationales alors partagées entre le musée de l'Homme et le Musée national des arts d'Afrique et d'Océanie. Le projet ambitionnait de revaloriser ces collections délaissées par le public en mettant en avant leur aspect esthétique et en leur accordant la même dignité que les chefsd'œuvre occidentaux. Le projet, soutenu par Jaques Chirac, était porté par Jacques Kerchache, grand collectionneur et marchand d'art, à l'initiative de l'entrée des arts africains, amérindiens et océaniens au Pavillon des sessions du musée du Louvre, inauguré en 2000 (L'Estoile 2007; Price 2007). En 1999, la réalisation du bâtiment, confiée à l'architecte Jean Nouvel, devint le grand projet culturel de la double présidence de Jacques Chirac.

La commande d'art aborigène commence au début de l'année 2002 lorsque Nouvel propose de faire intervenir un "groupe» de peintres aborigènes. Jacques Chirac soutient la mise en place d'un partenariat francoaustralien pour renforcer les liens politico-économiques entre les deux pays. L'Australian Council for the Art (ministère de la culture australien) co-organise le projet et place à sa tête les conservatrices aborigènes Hetty Perkins et Brenda Croft, respectivement conservatrices des départements d'art aborigène de l'Art Gallery of New South Wales (Sydney) et de la National Gallery of Australia (Canberra) ${ }^{3}$. Elles sont assistées par le cabinet d'architecture Craknell \& Lonergan désigné pour faire l'interface avec les équipes de Nouvel. Du côté français, c'est Philippe Peltier, responsable de l'unité patrimoniale de l'Océanie du musée, qui est en charge des opérations.

Le projet initial confie la réalisation d'un plafond à John Mawurndjul, célèbre peintre sur écorce, ayant déjà participé à une exposition à Mantes-laJolie en 2001, et descendant des peintres de Terre d'Arnhem avec qui avait travaillé Karel Kupka, anthropologue et artiste franco-tchèque dont les collectes de peintures sur écorce forment la principale collection australienne du musée du quai Branly (Peltier 1998; De Largy Healy 2010). Finalement, en mars 2004, le projet s'étend à huit artistes, quatre hommes et quatre femmes originaires de quatre grandes régions artistiques australiennes: Désert de l'Ouest, Terre
3. Les deux conservatrices n'en sont pas à leur première collaboration avec l'Australia Council for the Arts; elles avaient déjà organisé le pavillon australien de la Biennale de Venise en 1997, représenté cette année-là par trois artistes aborigènes : Emily Kame Kngwarreye, Judy Watson et Yvonne Koolmatrie. 


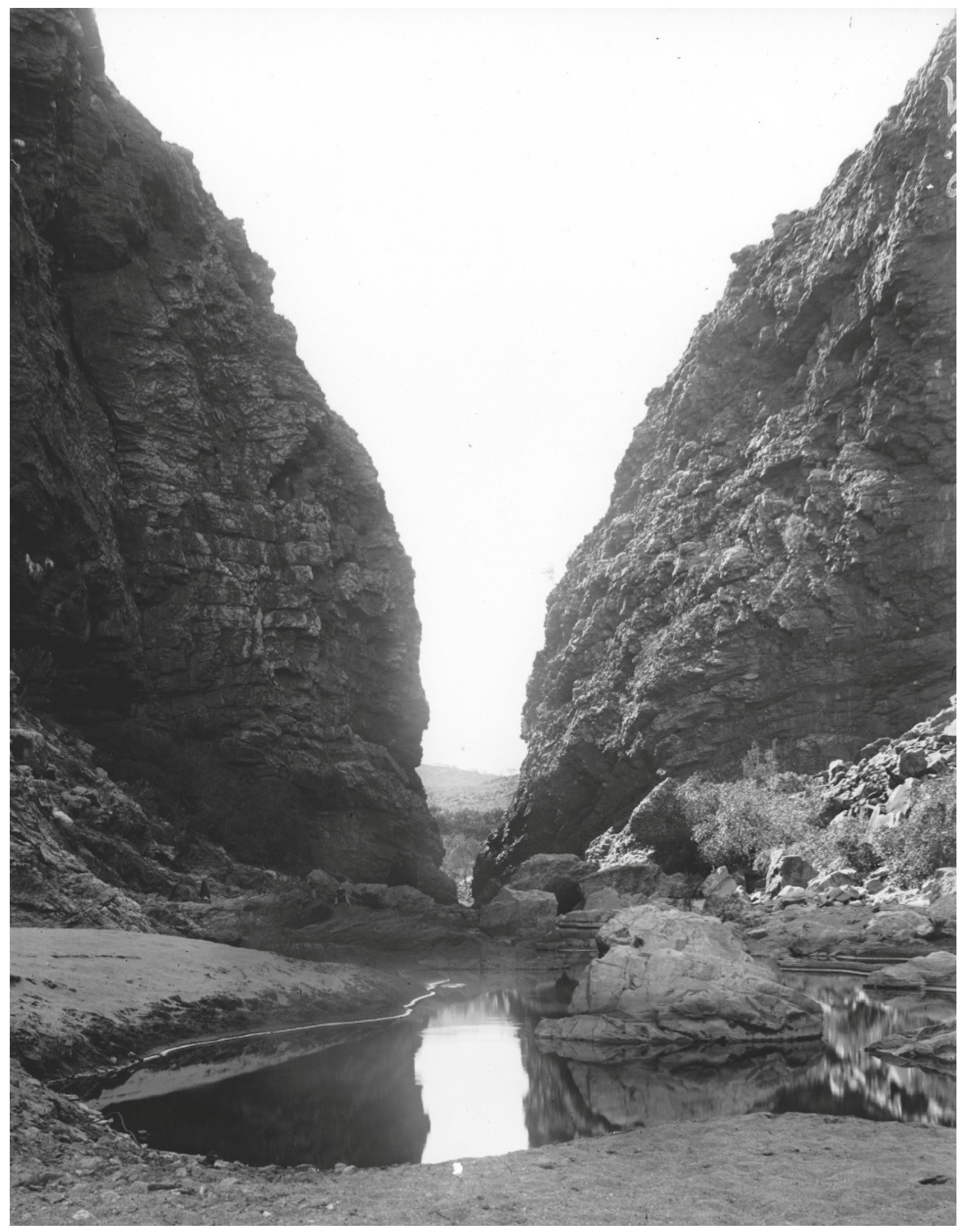


d'Arnhem, Kimberley et Sud-Est australien. II s'agit de Judy Watson (1959), Michael Riley (1960-2004), Ningura Napurrula (1938-2013), Tommy Watson (1935), John Mawurndjul (1952), Gulumbu Yunupingu (1943-2012), Lena Nyadbi (1936) et Paddy Nyunkuny Bedford (1922-2007). Est concerné l'ensemble du bâtiment administratif, un long bloc rectangulaire de cent mètres de long sur quinze de haut et huit de large, percé de fenêtres sur quatre niveaux. Dans un entretien donné au Financial Time, Brenda Croft affirme de façon provocatrice et humoristique: «On pourrait dire que nous recolonisons les Français ${ }^{4}$. 》

La plupart des pièces de la commande sont des adaptations d'œuvres originales préexistantes, choisies par les conservateurs dans les collections publiques australiennes, puis modifiées par les architectes pour être adaptées aux surfaces du bâtiment (murs, plafonds, fenêtres) en respectant la réglementation des bâtiments de France. Nouvel imagine un système de jeu de miroirs pour rendre visibles depuis la rue les peintures des plafonds et des murs intérieurs, tout en conservant une certaine discrétion. L'ensemble est ensuite validé ou modifié par les artistes, avant sa mise en œuvre par des équipes techniques australiennes et françaises. Différentes techniques sont utilisées, de la gravure sur verre à la peinture acrylique, en passant par la peinture aux pigments naturels, les gravures et peintures sur émail, ou encore la sculpture sur béton. Le résultat final relève ainsi d'une cocréation entre les artistes aborigènes, les équipes d'architectes françaises et australiennes, et les techniciens. Chaque étape de l'élaboration de la commande est contrôlée par les conservateurs et, dans une moindre mesure, par les artistes. Seul Mawurndjul vient réaliser sur place et superviser une partie de sa peinture sur le plafond de la librairie.

Le bâtiment laisse apparaître des horizons temporels différents, voire divergents: certaines œuvres montrent des types de cosmogonies, la création de lieux par des êtres totémiques, alors que d'autres font clairement référence à I'histoire coloniale. L'adaptation de l'œuvre de Napurrula (Wirrulnga, 2005) est ainsi constituée d'une accumulation de points blancs sur fond noir. Le tableau original évoque la faille rocheuse de Wirrulnga, au sud-est de la communauté Kiwirrkura, près de Mount Webb. L'endroit est un site sacré féminin lié à des rites de fécondité. La peinture de Tommy Watson, l'autre artiste du désert, repose sur des effets cinétiques qui rappellent visuellement certaines de nos images au télescope ou au microscope: une superposition de points colorés en strates plus ou moins denses dont les couleurs se révèlent en transparence par vagues, créant mouvement et vibration. Par là s'expriment les forces créatrices du paysage, les êtres totémiques dont les déplacements, les chants et les actions ont sculpté le monde. Par contraste, l'œuvre de Riley transcrite sur la façade, Dreamscape, est une série de sept photographies alignées montrant des objets-mémoires: aile d'oiseau, plume, insecte, Bible retournée, boomerang, vache et sculpture d'ange vue de dos sur un fond de ciel bleu ou nuageux. Une même séquence d'images réunit donc ici des références à l'imposition coloniale de la chrétienté et du pastoralisme qui ont entraîné la destruction des terres aborigènes et la dépendance alimentaire des populations, ainsi qu'à l'imaginaire du temps du rêve, concept propre à la temporalité aborigène. L'œuvre de Judy Watson traite, quant à elle, du document ethnographique dans son rapport à la mémoire. Elle a mené dans les années 1990 des recherches sur les objets aborigènes

\author{
4. Brenda Croft citée \\ par Jeremy Eccles, \\ in J. Eccles, «Aboriginal \\ Originals Woo French", \\ Financial Times, \\ 20 décembre 2004 (nous \\ traduisons). Voir aussi \\ Perkins et Croft 2006.
}

\author{
ci-contre \\ fig. 1 \\ Baldwin Spencer, \\ Expédition Horn. Simpsons \\ Gap (Arungachirpa ou \\ Urengetyerrpe), Territoire \\ du nord, Australie, vers \\ 1895, plaque de verre \\ (détail). Collection \\ Museums Victoria, \\ Melbourne (c) Museums \\ Victoria Collections.
}




\section{ci-contre}

fig. 2

Propulseur gravé en bois et pigments naturels, région du Kimberley, début $x x^{e}$ siècle. Certains propulseurs, utilisés comme objets rituels, comportent des gravures représentant des itinéraires mythiques, également présents sur les churinga (C) musée du quai Branly-Jacques Chirac. conservés dans les musées européens et reproduit dans ses carnets les formes de certaines pièces provenant des terres du groupe d'appartenance de sa mère dans le Queensland, notamment des colliers féminins en cheveux tressés. Dans certaines cultures aborigènes les cheveux renvoient à la transmission entre femmes mais aussi entre les vivants et les morts. Les dessins de Judy Watson intitulés Museum Piece 2006 ont été gravés à l'acide sur la vitre du rez-de-chaussée, en dessous des photographies de Riley. L'image de ces objets signale à la fois l'acte de dépossession coloniale à l'origine des collections ethnographiques et le geste de réapproriation mémorielle de l'artiste.

Selon des modalités différentes, les œuvres de la commande matérialisent ainsi des connexions temporelles similaires. Les artistes du désert comme Napurrula ou Tommy Watson ont recours à une iconographie traditionnelle (cercles, demi-cercles, lignes, points) pour convoquer dans le présent un temps ancien, alors que Riley et Judy Watson travaillent à partir d'un vocabulaire contemporain (installation, photographie, dessins figuratifs) pour interroger un passé fuyant, en lien avec la temporalité mythique aborigène. Dans les deux cas on retrouve aussi la rupture coloniale, marquant une discontinuité temporelle perceptible dans ces allers-retours entre passé et présent. La commande dans son ensemble est un acte de mémoire. En ce sens, le bâtiment administratif apparaît comme un monument, c'est-à-dire un lieu de remémoration, un assemblage de temps hétérogènes.

La contribution de Bedford à la commande est également traversée par cette dynamique. Son art est connu en Australie pour ses nombreuses références à l'histoire coloniale du Kimberley, tout en restant proche de certaines formes rituelles locales. L'examen de la trajectoire de son œuvre, de ses origines rituelles et des concepts temporels aborigènes permet de comprendre comment des temporalités multiples interagissent et s'incorporent dans ses peintures.

\section{Les origines rituelles de l'œuvre de Paddy Bedford}

L'œuvre de Bedford est sans doute la plus discrète de l'ensemble. Sur son côté ouest, le bâtiment est adossé à un immeuble haussmannien de l'avenue de La Bourdonnais. Les deux édifices sont séparés par un long passage vertical à ciel ouvert, rebaptisé « la faille» par les employés du musée et utilisé comme couloir d'approvisionnement, espace fumeur et entrée alternative par les visiteurs invités. C'est sur la paroi de ce couloir qu'a été reproduite l'œuvre de Bedford Thoowoonggoonarrin (2006). L'espace s'ouvre sur la rue de l'Université par une double porte coulissante transparente, laissant entrevoir la peinture selon un angle de $35^{\circ}$ depuis le trottoir. Elle occupe un rectangle de douze mètres cinquante de long sur cinq mètres soixante-quatre de haut, trouée dans sa partie supérieure d'une ouverture rectangulaire équipée d'une grille. Réalisée en pigments de silicate, elle figure une ligne horizontale noire rehaussée de points blancs qui divise la peinture en deux sections, l'une blanche sur la partie inférieure et l'autre noire sur la partie supérieure. D'un côté de la ligne, un amas de formes arrondies, deux blanches et une noire, de l'autre un cercle blanc, surligné de noir, petite sphère solitaire qui chevauche la ligne, répondant à la forme noire qui, en bas, semble sortir de terre. 


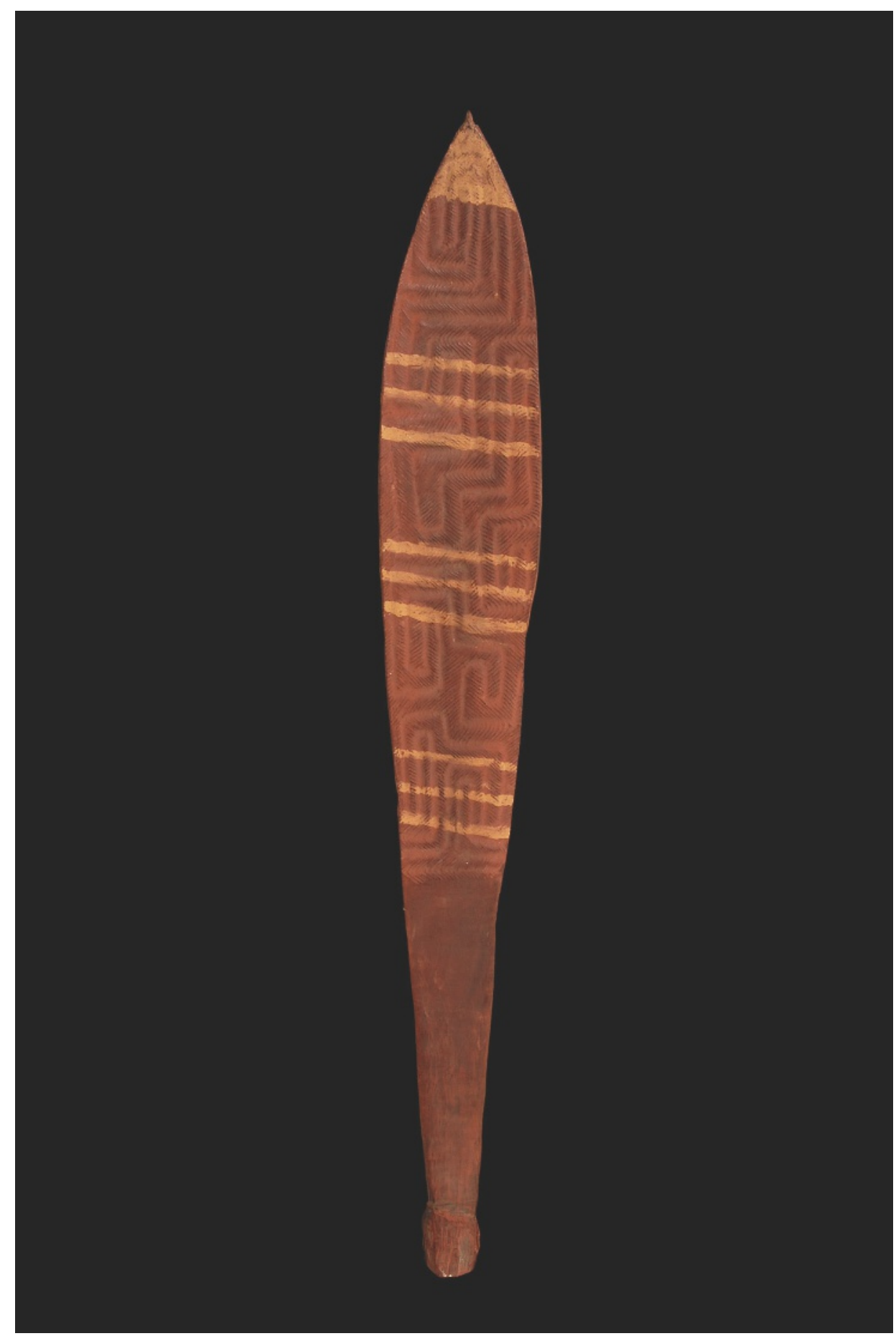




$$
0
$$




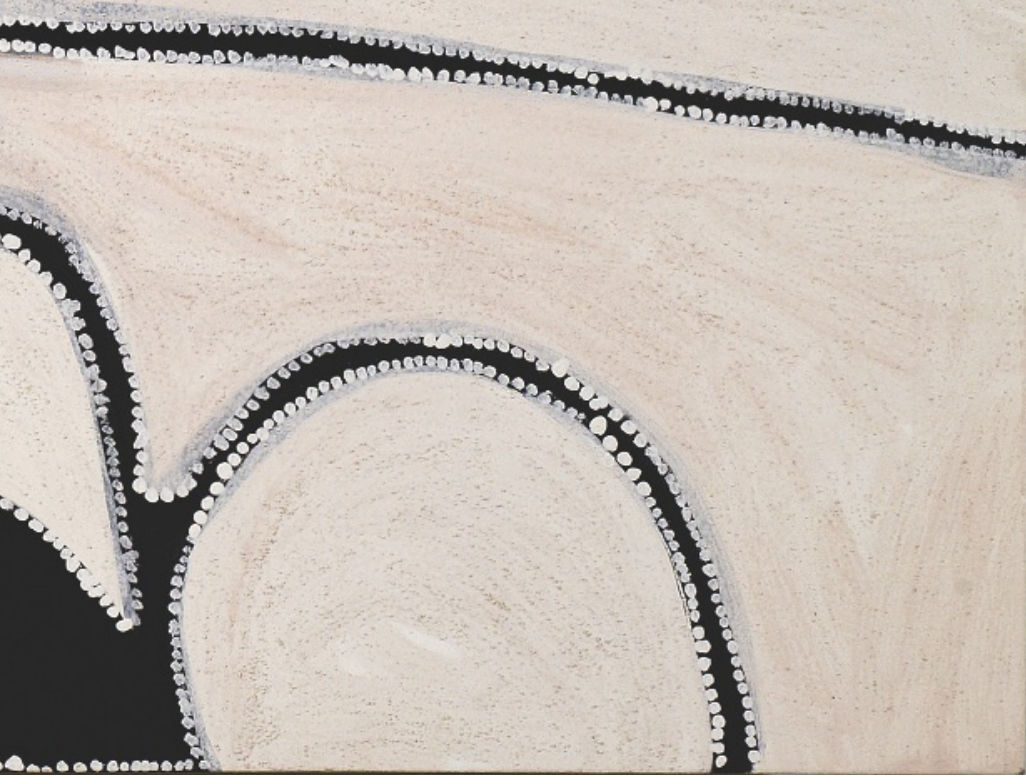

fig. 3

Paddy Bedford,

Thoowoonggoonarrin,

2006.

Pigments naturels (terre)

et liant synthétique sur lin, $150 \times 180 \mathrm{~cm}$.

Avec l'aimable autorisation

de Sotheby's Australia (c)

ADAGP, Paris 2018. 


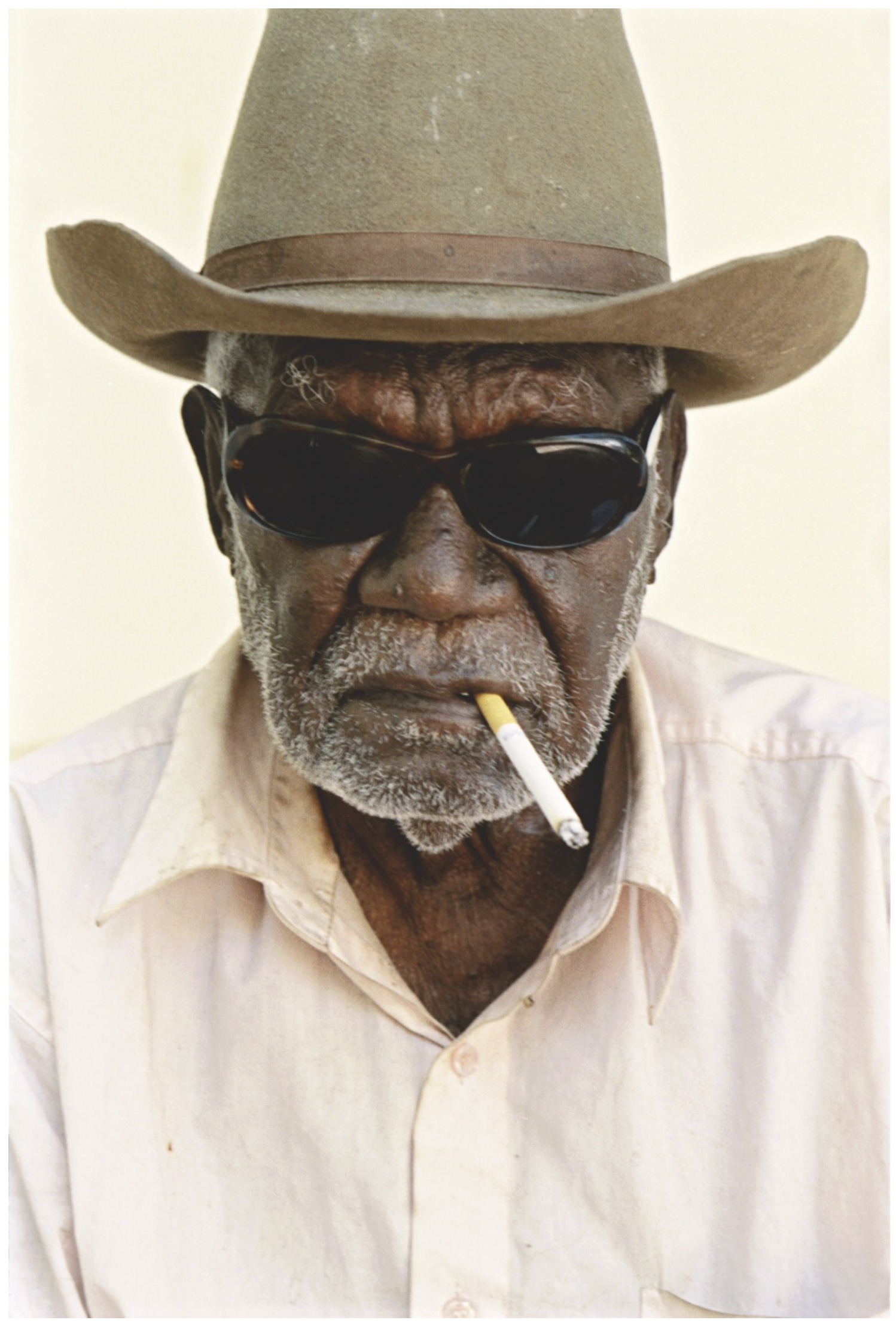


Thoowoonggoonarrin, que l'on peut traduire par «site du figuier », est une adaptation d'une peinture à l'ocre sur toile réalisée dans l'ancien studio de Jirrawun Arts 5 à Wyndham, dans le nord-ouest de l'Australie, en février 2006, soit quatre mois seulement avant l'ouverture du musée du quai Branly. L'œuvre originale est quasiment la seule peinture monochrome qu'ait jamais produite Bedford. Composition d'une grande sobriété, la toile se présente sur un fond blanc travaillé au gris à partir d'un mélange de pigments naturels. Dans l'installation du musée, l'effet de texture est resté fidèle à cette technique du wet on wet (mélange de couleur à même la toile), grâce à l'usage de silicate, pigment minéral de silice proche de ceux utilisés par Bedford.

La peinture originale est dérivée d'une tradition rituelle nommée balga (ou joonba) consistant à communiquer publiquement des images transmises en rêve par les esprits des morts (juwarri) en les matérialisant sous forme d'objets rituels utilisés comme support mnémonique. De même que la plupart des rituels kija visent à inscrire un événement dans un lieu, l'acte de peindre permet d'incorporer les événements dans des objets qui sont euxmêmes des images de lieux. C'est le peintre Rover Thomas, un ancien meneur de bétail d'origine kukadja, installé dans la communauté aborigène de Warmun, qui fut le premier à introduire l'utilisation de panneaux de bois pour réaliser des peintures d'un nouveau type à la fin des années 1970. L'esprit d'une vieille femme récemment décédée lui révéla en rêve une série de sites et d'événements signifiants qui reliaient plusieurs lieux formant un itinéraire. Les images et les chants qu'il reçut en rêve donnèrent lieu à une cérémonie nommée Gurirr Gurirr ${ }^{6}$. Le peintre transposa ses visions de manière semi-abstraite sur des panneaux qui attirèrent l'attention des anthropologues et des musées, puis des collectionneurs ${ }^{7}$ (Stanton 1986; Thomas et al. 1994; Macha et McLeod 2003). En réponse à cette demande, les peintres du Gurirr Gurirr comme Thomas et Paddy Jaminji commencèrent à produire de nouvelles peintures sur bois et sur toile dont les sujets ont rapidement dépassé les thèmes de la cérémonie initiale. Un petit groupe d'artiste se mit à représenter ses propres terres et les histoires qui y étaient associées, initiant un véritable mouvement artistique.

L'une des innovations picturales de Thomas, reprise par Bedford, est d'avoir transposé ses visions sous forme de paysages, une sorte de cartographie des lieux traversés par les esprits, comme flottant au-dessus de la surface $^{8}$. Ce type de représentation était jusqu'alors inexistant chez les groupes du Kimberley oriental qui utilisaient dans leurs rituels des woorrangoo, structures tissées en trois dimensions que les danseurs portaient sur les épaules. La surface en bois de ces objets fut alors élargie pour en faire de véritables panneaux rituels portés, telles des fenêtres ouvertes sur la vision des esprits. Privilégiant de larges aplats d'ocres aux textures mates ou translucides, les peintures évoquent de manière minimaliste les traces du passage des ancêtres mythiques ayant modelé les paysages. Le paysage n'est pas perçu selon un point de vue latéral fixe, tel qu'il s'offre au regard, mais prend la forme d'une représentation transversale. La référence visuelle n'est pas une ligne d'horizon ou un point de fuite, mais la surface du sol, conçue comme une membrane perméable entre le dessus et le dessous, l'intérieur et l'extérieur. Les peintres du Kimberley donnent à voir la structure interne des éléments topographiques comme passés au rayon $X$, dévoilant une géographie invisible à l'œil nu (Ryan 1993; Dolk 2006; Morvan 2012).
5. Créé en 1998 par Freddie Timms et Tony Oliver, Jirrawun Arts était une entreprise aborigène représentant un groupe d'artistes de langue kija dont Paddy Bedford était l'un des membres fondateurs.

\section{Cette cérémonie a été interprétée par l'anthropologue Kim Akerman comme faisant partie d'un mouvement local de renouveau culturel aborigène à la fin des années 1970 avec, dans plusieurs régions, la reprise d'initiations interrompues au début du siècle (Akerman 1980).}

7. Les plus anciens panneaux du Gurirr Gurirr sont aujourd'hui au Berndt Museum, le musée de I'université d'Australie occidentale à Perth.

8. Cette vision traduit l'influence des cultures aborigènes du désert dont les peintures sur le corps ou sur le sol s'apparentent à des cartes topographiques.

\section{ci-contre}

fig. 4

Katie Wilson,

Portrait de Paddy Bedford vers 2002 @ Katie Wilson. 
9. Le catalogue de la commande publique d'art aborigène australien parle ainsi de "culture éternelle" transposée dans un

"vocabulaire contemporain" (Australia Council for the Arts 2006: 7). Le Figaro Magazine du 24 juin 2006 a publié un reportage sur la rencontre avec Tommy Watson dans le centre de l'Australie: "Les peintres aborigènes nous propulsent au

"Temps du rêve", celui de la création du monde par les êtres ancestraux. [...] Tommy Watson montre une totale indifférence à ce qui l'entoure. Assis sur sa toile, il pose un point puis un autre. [...] II semble guidé par une force lui imposant couleurs, composition, modelé de la touche."
Les marques liées à la colonisation ne sont pas non plus absentes: clôtures, routes, ponts, chemins de fer, sites funéraires, emplacements des massacres, rythment les paysages sous forme de lignes ou de figures semiabstraites. Chez Bedford par exemple, notamment dans une série de peintures réalisées entre 1999 et 2005, les cercles rouges surlignés de points blancs sont les traces d'un massacre ayant eu lieu près de Bedford Downs dans les années 1920. Le tableau Emu Dreaming (2003) de la National Gallery of Australia, initialement sélectionné pour la commande du musée du quai Branly, représentait la juxtaposition entre le site du massacre et l'emplacement d'un lieu sacré à Mount King près de Bedford Downs. Ce projet dut être abandonné en raisons de contraintes techniques quelques mois avant l'ouverture du musée.

Les peintures de Bedford constituent ainsi des supports mnémoniques qui matérialisent, sous formes d'empreintes ou de traces, des rapports entre des événements issus de différentes temporalités. Le mouvement, le rythme et l'instabilité des peintures sont un indice de leur nature performative comme paysages mouvants suspendus au-dessus de la tête des danseurs. Par effet de miroir, les peintures montrent l'inscription des corps à la surface du sol, les points sur la toile faisant écho aux pas des danseurs comme image de la temporalité humaine dans les paysages ancestraux (Morvan 2014). Cette juxtaposition temporelle se retrouve dans Thoowoonggoonarrin, comme le suggèrent le cartel et la notice affichés dans le couloir et du côté de la rue de l'Université:

Paddy Nyunkuny Bedford, C. 1922/Gija/East Kimberley, Western Australia, Thoowoonggoonarrin, 2006. Paddy Bedford est né à Bedford Downs Station, dans l'est du Kimberley. Depuis son plus jeune âge, Paddy Bedford a mené de front son travail de gardien de troupeaux et son apprentissage des coutumes traditionnelles du peuple Gija. En 1998, il commence à peindre au sein de la coopérative Jirrawun Aboriginal Arts. L'œuvre de Paddy Bedford dévoile une connaissance intime de sa terre d'origine et des traditions et croyances religieuses du Ngarranggarni. Les histoires ancestrales du peuple Gija en font partie, tout comme les événements d'aujourd'hui. Cette œuvre fait référence à un figuier qui pousse dans un enclos à bétail de la région d'origine de sa mère.

La référence au concept de Ngarranggarni est ici problématique car elle semble suggérer une équivalence entre les récits ancestraux et les événements contemporains sans expliciter les liens temporels qui les unissent. La plupart des documents accompagnant la commande, élaborés par l'Australian Council for the Art, présentent l'art aborigène comme étant à la fois traditionnel et moderne, sans préciser la nature de ce rapprochement. La réception médiatique de la commande reprend le même discours ${ }^{9}$. La répétition d'une rhétorique de la symétrie entre l'ancien et le nouveau, le «sans âge » et le «contemporain », couplée à l'absence d'information sur les références historiques contenues dans certaines œuvres, provoquent une sorte d'aplanissement temporel qui rend difficile toute analyse diachronique des peintures. Pourtant, l'articulation des événements mythiques et historiques est au cœur de la dynamique temporelle de la commande et des œuvres de Bedford. 


\section{Dreamtime et discontinuités temporelles}

Le concept de dreamtime ou «temps du rêve», fréquemment décrit comme un «passé mythique de la création du monde», condense à lui seul la difficulté d'accès à la temporalité particulière des Aborigènes (Wolfe 1991; Swain 1993; Charlesworth, Dussart et Morphy [éd.] 2005). C'est Spencer, professeur de biologie à l'université de Melbourne, et Gillen, responsable de la station de télégraphe d'Alice Springs, qui forgent le terme dreamtime à la suite de l'expédition scientifique Horn de 1894 en Australie-Centrale. L'association entre les deux hommes donna naissance au premier ouvrage ethnographique détaillé sur les groupes aborigènes du centre, Native Tribes of Central Australia (2008 [1899]). L'expression dreamtime apparaît dès 1896 dans le rapport de l'expédition. Pour Spencer, l'expression correspond à la traduction du nom donné à un passé très reculé dans lequel les ancêtres mythiques de la tribu sont supposés avoir vécu, les Aborigènes se considérant en effet comme les descendants d'êtres totémiques hybrides à l'origine des paysages, des espèces et des lois. Une fois leurs actions ordonnatrices effectuées, ceux-ci sont retournés sous terre, et certaines parties de leur corps se sont transformées en éléments du paysage: rochers, failles, collines, sources, arbres, etc.

La notion de temps du rêve était liée dans l'esprit de Spencer aux conceptions anthropologiques de son époque, et en particulier à l'évolutionnisme de Edward Tylor dans son ouvrage fondateur Primitive Culture, paru en 1871. Pour Tylor, la «culture primitive» ne distinguait pas nettement le rêve de la réalité, ni la magie de la science. En quelques années, le concept de dreamtime et le totémisme se retrouvent à la base des grandes théories constitutives des sciences de l'homme du début du xx siècle: l'anthropologie avec James Frazer dans Totemism and Exogamy (1910), la psychanalyse de Sigmund Freud dans Totem et Tabou (1912-1913) et la sociologie avec Émile Durkheim dans Les Formes élémentaires de la vie religieuse (1912).

Dans l'ouvrage de 1899, Spencer reconnaît que le terme Alcheringa recoupe, d'après la tradition aranda, au moins «quatre périodes plus ou moins distinctes ", chacune marquée par un événement: la création des hommes et des femmes, l'introduction du rite de circoncision, puis du rite de sub-incision, enfin l'institution du système de mariage. II précise que « la seconde et la troisième périodes ne sont pas cependant, loin s'en faut, clairement définies, et sont dans une certaine mesure contemporaines l'une de l'autre, ou plutôt entrecroisée 10 " (Spencer et Gillen 2008 [1899]: 362). Curieusement, cette «épaisseur» temporelle ne sera reprise ni par les intellectuels de l'époque ni par leurs successeurs. Adolphus Elkin traduisit alcheringa par ever-present, un «présent perpétuel ». Radcliffe-Brown parle quant à lui de world-dawn ou «aube du monde». Qu'il se réfère à un passé éternel ou à un perpétuel présent, le concept suggère chez ces auteurs un amalgame entre le passé et le présent, un effacement de la durée au profit de la permanence (Radcliffe-Brown 1931; Elkin 1933).

Dans une série d'articles publiée par la revue Océania entre 1959 et 1963, l'anthropologue australien William Edward Hanley Stanner adopte, contre le fonctionnalisme dominant, une approche phénoménologique du dreamtime qu'il appelle dreaming (Stanner 1989 [1959]; Keen 2005). II fut le premier à considérer le dreaming comme principe central des religions aborigènes et non comme fonction annexe de l'organisation totémique, ses analyses ouvrant ainsi la voie à des recherches approfondies sur les complexes mythico-rituels australiens:
10. Nous traduisons.

Carl Strehlow, missionnaire et linguiste à Hermansburg, au sud d'Alice Springs, réfute dès le départ la traduction de Alcheringa par dreamtime. Pour Strehlow, la racine altjira (le "alchera " dans Alcheringa) n'a en elle-même pas de sens tandis que sa forme verbale altjirerama signifie « devenir dieux " (Strehlow in Thomas 1905: 403). 
11. Nous traduisons.

\section{ci-contre}

fig. 5

Anonyme,

Paddy Bedford travaillant sur Emu Dreaming, 2003.

Peinture représentant

l'emplacement du massacre

de Bedford Downs. Archive

de Jirrawun Arts. Droits

réservés (C) ADAGP.

Paris 2018.
Le Dreaming évoque une notion de temps sacré légendaire issue d'un passé infiniment reculé qui, dans un certains sens, fait toujours partie du présent. On ne peut pas fixer le Dreaming dans le temps: il a été et demeure everywhen («partout-et-tout-le-temps»). [...] C'est par l'acte de rêver, en tant que symbole et réalité, que l'esprit aborigène établit des contacts - pense qu'il établit des contacts - avec quelques éléments mystérieux qui connectent le Dreaming à l'ici et maintenant ${ }^{11}$. (Stanner 2009: 58)

À travers le néologisme everywhen, la définition de Stanner pointe la relation du temps à l'espace dans le concept de dreaming. II désigne également l'activité onirique comme opérant une connexion entre un passé mythique et un présent spatio-temporel. Mais tout en affirmant l'impossibilité de fixer le dreaming dans le temps, et en insistant sur son caractère omniprésent et permanent (partout et tout le temps), cette définition ne permet pas de comprendre les dynamiques temporelles hétérogènes à l'œuvre dans le dreaming. L'insistance sur la permanence suggère une forme de continuité du temps des origines dans le présent qui empêche de saisir les ruptures.

C'est en se tournant vers l'étude précise des rituels warlpiri que les recherches de Barbara Glowczewski ont permis de mieux cerner les dynamiques temporelles dans le concept de dreaming (jukurrpa en warlpiri). Elle définit le jukurrpa non pas comme une période identifiée au passé ou au présent mais plutôt comme une mémoire déployée dans l'espace. Cet espace-temps produit par les êtres totémiques est accessible aux humains via les activités oniriques et les performances rituelles. Cette mémoire est disséminée dans les lieux de passage des ancêtres totémiques, conçus comme des puits temporels reliés entre eux pour former un vaste réseau, de sorte qu'il n'y a pas de temps en soi mais seulement des temps hétérogènes, localisés et interconnectés (Glowczewski 1991 et 2015). Le caractère dynamique de cette mémoire inscrite dans la terre repose sur le statut singulier des rêves dans les cultures aborigènes: la capacité de chaque individu à transformer la mémoire du dreaming grâce aux révélations véhiculées par les rêves nocturnes. Les informations transmises en rêve sont conçues comme des réminiscences d'événements oubliés qui peuvent venir modifier, prolonger et transformer le corpus mythique existant ou, dans certains cas, révéler des épisodes historiques inconnus. Certains individus connaissent des épisodes de révélation nocturne pendant lesquels un être totémique les contacte pour leur montrer un événement, un lieu ou une cérémonie jusqu'alors inconnus. Lorsqu'un tel rêve se produit, il initie un long processus d'interprétation collective entre le rêveur et les autres membres de la communauté, à la suite duquel les connaissances révélées sont éventuellement intégrées au corpus existant et donnent lieu à de nouvelles cérémonies.

\section{Les morts et l'inscription de l'histoire dans le paysage}

Qu'en est-il des "événements contemporains" également englobés dans le Ngarranggarni dont parle la notice de Thoowoonggoonarrin? Ceux-ci, dans leur accumulation, peuvent-ils rendre compte d'une profondeur historique, d'une suite ordonnée d'événements? On ne prête souvent aucune conscience historique aux Aborigènes réputés pour leur ontologie exclusivement spatiale (Swain 1993) ou leur façon de diluer l'événement humain dans 


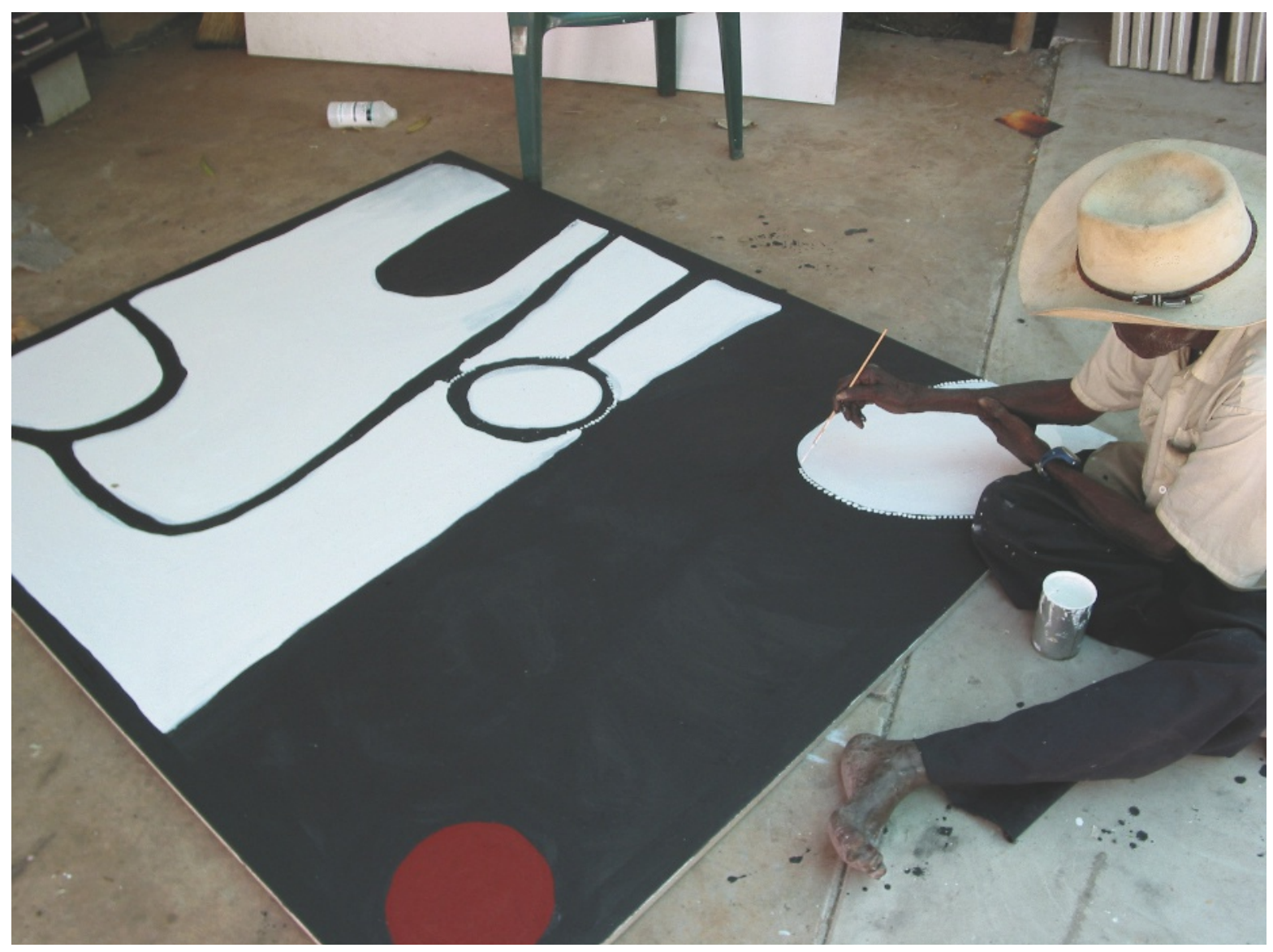

l'ordre cosmique du dreamtime provoquant un «effacement de l'historique» (Myers 1986). Pour l'anthropologue Alan Rusmey, au contraire, il existe un «mode d'inscription» de l'histoire aborigène dans les paysages: «C'est en réalité un mode d'orientation beaucoup plus global, à travers lequel une part considérable de ce que nous appelons "l'histoire" - en tant qu'actions passées de personnes connues et nommées - est inscrite et se retrouve dans le paysage 12. (Rumsey 1994: 116)

Dans le Kimberley, le processus de révélation onirique ne se produit pas par le biais des êtres totémiques, mais par celui des morts qui entrent dans les rêves des vivants et transmettent des messages issus du dreaming. Les esprits des morts jouent un rôle d'intermédiaire entre la mémoire mythique des êtres totémiques et la mémoire historique humaine. Les morts appelés juwarri sont des esprits n'ayant pu regagner leur centre totémique pour retourner à l'état de marlinbany («esprit-enfant ${ }^{13}$ ») dans l'attente d'une nouvelle incarnation. Ils errent aux abords du lieu de leur décès ou de leurs sépultures et entrent parfois en contact avec les vivants ${ }^{\mathbf{1 4}}$. Les parents identifient régulièrement leur nouveau-né à des personnes décédées plus ou moins récemment (Kofod 2002 et 2006; McDonald 2010). Bedford lui-même est reconnu par ses pairs comme étant la réincarnation d'un vieil homme décédé à Violet Valley peu avant sa naissance. On peut ainsi parler de généalogie «anachronique», car les morts et leur passé resurgissent parmi les vivants.
12. Nous traduisons.

13. Ces esprits-enfants sont qualifiés de marlinbany (littéralement " qui ont des herbes vertes") et sont associés aux algues qui poussent au fond des points d'eau, l'esprit marlinbany se frayant un chemin dans le ventre de sa future mère lorsque celle-ci passe à proximité (Kaberry 1939: 46).

14. D'autres cherchent à entrer en contact avec un clever man (sorcier) guérisseur) avec qui ils communiquent directement (McDonald 2010: 58-59). 


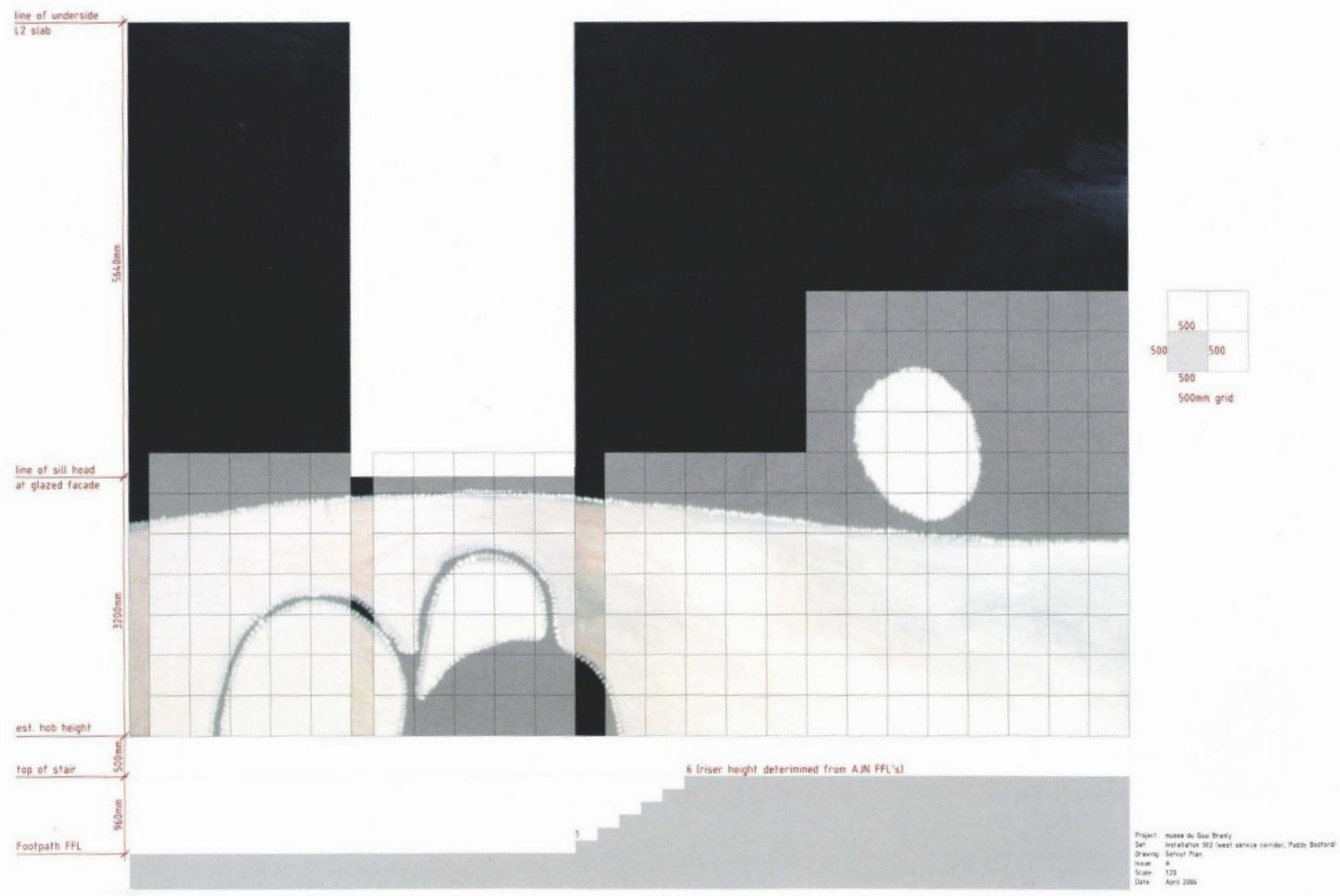

15. Le mort retourne à l'état de totem et disparaît peu à peu de la mémoire des vivants jusqu'à sa prochaine incarnation. Lorsqu'il est incapable de réintégrer son site totémique, il devient un juwarri et entre en contact avec les vivants, en portant avec lui la mémoire de son existence passée.

\section{ci-dessus}

fig. 6

Projet initial de la

commande d'art aborigène du musée du quai Branly (plan de la façade), 2005 Avec l'aimable autorisation de Cracknell \& Lonergan Architects Pty Ltd Newtown, Australie.
De ce fait, les événements liés aux vies antérieures des morts sont toujours présents dans la temporalité humaine (Morvan 2010). En refusant de mourir, les juwarri instaurent une discontinuité entre le temps mythique et le temps historique ${ }^{15}$.

Le statut même du mort et du cadavre chez les Kija comporte une composante temporelle importante. Les rites funéraires consistent à inscrire les morts à la fois dans le paysage et dans la mémoire du dreaming. Au terme d'une cérémonie qui s'étend sur plusieurs années, les os du défunt sont recueillis, nettoyés et enduits d'ocre avant d'être enroulés dans une écorce souple. Cette enveloppe (moonda) est ensuite placée sur une hauteur, généralement dans une faille ou dans le renfoncement rocheux d'une colline. Puis une série de chants et de danses vont réunifier la personne et son totem pour assurer le retour de l'esprit vers son centre totémique. La mort n'est donc pas une abstraction mais une présence qui doit être contenue dans des lieux (Harrison 2003; Langton 2005).

Les rencontres avec les esprits des morts donnent souvent lieu à une transmission de chants et d'images qui se matérialisent dans des rituels et des objets. Outre la révélation de nouveaux épisodes mythiques ou de sites totémiques, les juwarri communiquent souvent des événements de leur vie antérieure, parfois en rapport à leur décès. Dans le Kimberley, de nombreux événements historiques liés à la colonisation ont ainsi été incorporés à l'activité rituelle (Morvan 2010). L'œuvre de Bedford prend sens dans le cadre de la cérémonie Marnem dililib benuwarrenji (aussi appelée «massacre joonba») 
associée au massacre de Bedford Downs survenu dans les années 1920 alors que les conflits de frontière était particulièrement meurtriers. Les chants, les danses et les objets rituels manipulés pendant cette cérémonie (structures tressées woorrangoo, panneaux peints, bâtons de danse, masques) retranscrivent en détail cet événement tragique. $Y$ sont évoqués les conditions de vie des travailleurs aborigènes dans les fermes d'élevage au début du $x x^{e}$ siècle, les incidents ayant mené au massacre, l'empoisonnement des Aborigènes par les fermiers, la crémation des corps dans un bûcher, la transformation des morts en esprits juwarri, le voyage post mortem des esprits dans le but de trouver un chamane capable de capter et de traduire leurs chants. Plutôt que de faire l'objet d'une narration, le massacre est encodé sous forme rituelle, se manifestant dans et par le corps des participants. D'après sa gardienne actuelle, cette cérémonie ou joonba a été «trouvée» par un chamane de langue worla près de Kimberley Downs, soit à plusieurs centaines kilomètres du site du massacre. II a ensuite circulé entre plusieurs groupes de l'ouest du Kimberley, notamment chez les Ngarynyin et les Unambal, et été remis à un groupe d'hommes Kija au milieu des années 1930 lors d'un voyage d'initiation auquel participaient les jeunes Timmy Timms et Bedford (Morvan 2010 et 2014). Le joonba a opéré un retour dans son lieu d'origine, à Bedford Downs, et fut produit secrètement tout au long des années 1940 dans les différentes fermes de la région où résidaient les familles des victimes du massacre. Après une longue période de latence et d'oubli, le joonba fut ravivé (par Timms et Bedford) au début des années 2000 pour donner lieu à une série de peintures cartographiant les différents sites de massacres de la région ${ }^{16}$.

L'événement, qu'il soit historique ou mythique, n'est donc pas figé dans le passé, mais en dialogue avec le présent où il est constamment réactivé à la manière d'une onde ou d'une radiation, en fonction de contingences politiques ou sociales. Le temps long et géologique des transformations des paysages par les êtres totémiques croise le temps bref et historique des actions humaines, tous deux articulés par la temporalité du dreaming qui crée des ponts entre des événements chronologiquement éloignés. Événements mythiques et historiques se superposent en un même lieu, créant une profondeur temporelle, un passé organisé en paliers discontinus. Le dreaming agit comme une machine à faire émerger des événements oubliés et à articuler des temps discordants. Ce processus s'incorpore dans les rituels et les peintures, y compris celles destinées aux musées et aux collectionneurs.

\section{Thoowoonggoonarrin ou la faille temporelle du musée du quai Branly}

Né en 1922 sur les terres du ranch de Bedford Downs, Bedford a travaillé une grande partie de sa vie dans les fermes d'élevage, avant d'occuper différents emplois dans la construction des routes et des ponts qui accompagnaient le développement de l'industrie bovine dans la région. Le Kimberley fut colonisé dès les années 1880, lorsque de grands propriétaires du Queensland envoyèrent leurs troupeaux dans l'ouest à la recherche de nouveaux pâturages à exploiter. Ils prirent de fait possession des terres aborigènes et vinrent rapidement à bout des mouvements de résistance locaux. Perpétrés par la police et les colons, des dizaines de massacres, souvent non répertoriés par les autorités, eurent lieu dans tout le Kimberley. La situation se stabilisa dans les années 1930 avec l'installation de nombreuses familles autochtones
16. La première

performance eut lieu devant la principale poste de police de la région, en signe de réconciliation après des incidents ayant opposé les forces de l'ordre et des jeunes Kija. Le retour de cette cérémonie et les peintures qui

l'accompagnèrent donnèrent lieu à de vifs débats sur la place de la mémoire aborigène dans I'histoire coloniale australienne (Langton, Oliver et Kofod 2002; Manne 2003 ; Préaud 2007 ; Morvan 2010). 
17. Entretien réalisé par l'auteur en avril 2006. Paddy Bedford s'exprime dans un mélange de kimberley kriol (pidgin) et de kija. La transcription contient à la fois les mots de Bedford et leur traduction par Frances Kofod pour les passages que je n'ai pu comprendre.

\section{ci-contre}

fig. 7

Musée du quai Branly-

Jacques Chirac, bâtiment Université, rez-de-chaussée. Vue intérieure avec l'œuvre de Paddy Bedford, Thoowoonggoonarrin 2006 @ musée du quai Branly - Jacques Chirac, photo Nicolas Borel (c) ADAGP, Paris 2012. autour des fermes où les hommes étaient utilisés comme main-d'œuvre semi-permanente contre de maigres rations alimentaires et du tabac, tout en ayant la possibilité de rester à proximité de leurs terres.

Dans sa jeunesse, Bedford fut emmené à la léproserie de Derby à six cents kilomètres au sud-ouest du Kimberley, où il noua des liens étroits avec les groupes aborigènes locaux qu'il conserva toute sa vie. Au fil des initiations, ses responsabilités dans la sphère religieuse kija et les alliances qui le maintenaient en contact avec les Aborigènes de Derby lui octroyèrent le statut d'homme initié de haut degré (manambarrany). Cependant, suite à un accident survenu à la fin de sa carrière, il se retrouva à Warmun sans emploi et dans une relative pauvreté. Au milieu des années 1990, il se mit à peindre sur des chutes de bois, en empruntant le style de Thomas récemment décédé et en s'inspirant des motifs réalisés sur les corps ou sur différents objets pendant les cérémonies. Bedford rencontra le galériste Anthony Oliver, venu de Melbourne dans le Kimberley à la demande de son neveu, Freddie Timms, qui avait créé le groupe d'artistes de Jirrawun Arts en 1998. Sous la houlette de Oliver, le vieil initié kija développa un expressionnisme qui tirait l'interprétation topographique des paysages du Kimberley vers une abstraction minimaliste. En l'espace de cinq ans, Bedford devint l'un des artistes australiens les plus cotés sur le marché de l'art.

Au milieu des années 2000, en raison de son succès fulgurant, sa parentèle élargie exerça sur lui une pression financière grandissante. II s'était éloigné de Warmun pour résider dans la petite ville de Kununurra (à plus de deux cents kilomètres), dans la résidence de la linguiste Frances Kofod, dont il occupait la terrasse surélevée de manière quasi permanente. Malgré cet éloignement du pays kija, ses revenus conséquents lui permettaient d'effectuer des voyages réguliers sur ses terres autour de Bedford Downs. De retour de ces voyages, il se plongeait dans un état méditatif prolongé, parfois pendant plusieurs jours. Je le côtoyais alors quotidiennement, et il n'était pas rare de le voir passer de longs moments seul, à la tombée de la nuit, observer la montagne, chantant quelques vers à voix basse, ou s'adressant à des personnes invisibles, traversant la frontière toujours poreuse pour les Kija entre le monde des vivants et celui des esprits. II me confia que les esprits des anciens habitants de Bedford Downs revenaient lui parler en rêve. Des épisodes, parfois macabres, du passé de sa famille revenaient ainsi à sa mémoire. C'est dans ce contexte que Bedford entreprit en février 2006, au cœur de la saison des pluies, Thoowoonggoonarrin, l'œuvre exposée au musée du quai Branly. Lors d'un entretien, quelques semaines avant l'ouverture du musée, il m'expliqua pour la première fois les références temporelles de sa peinture ${ }^{17}$ :

Thoowoonggoonarrin, on l'appelle, je ne sais pas comment les blancs le nomment. Ça vient de Thoowoonggoony («figuier»), c'est juste le nom de cet endroit. Ce gros arbre se dresse quelque part sur cette colline. C'est sur les terres de ma mère, du côté de son frère. Il y a de l'eau dans un réservoir derrière. Et il y a un portail par où l'on faisait passer le bétail. Cette peinture vient de Bedford Downs.

[À propos des formes arrondies dans la peinture]. C'est de l'eau pour le bétail. Et la route pour les y amener. L'arbre Thoowoonggoony se trouve là. Quand j'étais enfant, on me portait sur les épaules, on marchait tout du long... Ensuite, je suis monté à cheval, partout. 


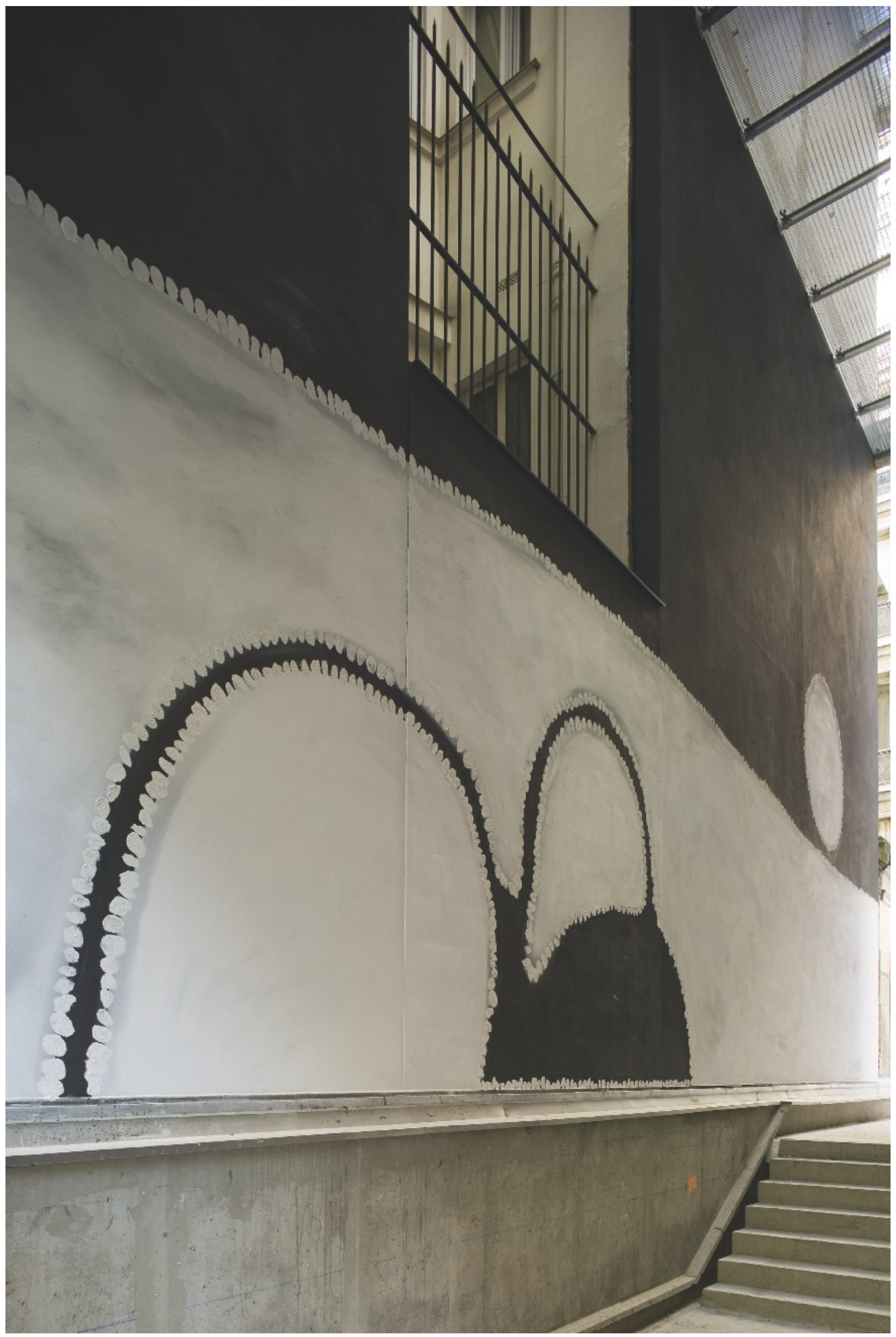


18. De 1936 à 1967 le gouvernement australien retirait systématiquement les enfants métis à leurs parents aborigènes pour les placer dans des institutions blanches. Les naissances étaient souvent issues de viols, et les enfants n'étaient pas toujours bien accueillis. Certains Aborigènes cachaient leurs enfants métis en les enduisant de charbon pour ne pas se les voir retirer, mais des cas d'infanticides furent aussi enregistrés.
Bedford évoque ensuite la sœur de sa mère dont la sépulture est située près de Thoowoonggoonarrin:

La sœur de ma mère, la plus jeune. Je ne sais pas ce qu'il s'est passé mais ils l'ont tuée pour rien. Des Aborigènes (blackfellow). Elle est tombée malade et elle est morte. On l'a mise en haut, dans un arbre. Ils ont mis le corps sur une boondoorra (plate-forme en bois), sur ces terres, sur le flanc de la colline. Ensuite ils l'ont récupérée et placée dans une grotte. Ils l'ont coupée et enveloppée dans une moonda («écorce»). Ils lui ont fait une couverture avec le moonda et l'ont mise dans cette grotte ici. [Montrant la peinture] Tout cela c'est mon pays. La mère de ma sœur est morte, c'est terminé. Tous les miens sont partis maintenant, je n'ai plus personne. Ils sont tous morts.

Toujours en regardant sa peinture, Bedford parle d'Old Bedford, la première ferme construite sur les terres de Bedford Downs. Sa mère y avait travaillé lorsqu'elle était jeune et était tombée enceinte d'un homme blanc ${ }^{18}$ :

C'est Old Bedford, là, à côté de Mendoowoorrji. Ma mère y travaillait quand elle était jeune. Elle a eu un kardiya («blanc»), un petit enfant kardiya. II est né là-bas, tu vois. Ensuite, elle a été envoyée à Moola Bullla, une ferme du gouvernement (qui faisait office de camps de travail). Ils l'ont tué ce gamin blanc, il était déjà grand, il a été noyé. Avant ma naissance... Et ce kardiya est né là-bas à Old Bedford. Parfois je rêve que je me dispute avec ce petit garçon qui a été noyé [...]. Elle ne l'a pas laissé grandir. Tout ce pays, c'était des terres aborigènes à l'époque, avant que les blancs s'en emparent.

L'enfant métis, désigné par l'expression little kardiya («petit blanc»), est né à Bedford plusieurs années avant Bedford et a été noyé quelque temps plus tard. C'est dans le réservoir derrière l'enclos à bétail de Thoowoonggoonarrin que l'enfant a trouvé la mort. À la suite de cet événement, sa mère fut envoyée dans le camp de travail de Moola Bulla près de la ville minière de Halls Creek. Depuis un récent voyage en hélicoptère à cet endroit, Bedford reçoit des visites de l'esprit de ce petit frère blanc qui revient pour le tourmenter. Les deux frères auraient de violentes disputes en rêve.

L'entretien permet d'isoler des éléments signifiants: un point d'eau, une route, un enclos à bétail, un figuier et la marque de deux morts dans le paysage. Bedford explicite d'abord le titre de la peinture, nommée d'après un type de figuier qui pousse à flanc de colline mais dont la présence semble anecdotique. Puis il attire mon attention sur un point d'eau, le réservoir pour le bétail, une route qui traverse les lieux, des souvenirs d'enfance de voyages à pied avec les membres de sa famille, la présence d'un site funéraire dans le paysage où repose une parente décédée (sa tante). Bedford désigne la forme arrondie (dans la partie supérieure de la toile) comme le point d'eau, et la ligne horizontale comme la route empruntée par le bétail pour rallier Old Bedford.

En consultant une carte de la région, on s'aperçoit que l'endroit (Thoowoonggoonarrin) est traversé par une route qui suit une rivière nommée Tugunari au sud de Bedford Downs; celle-ci serpente entre des collines et des amas rocheux qui s'apparentent parfois à des failles ou des petits canyons. Les trois formes arrondies du bas de la peinture, deux blanches 
et une noire, matérialisent certainement les collines et l'amas rocheux de Thoowoonggoonarrin. La forme noire représente sans doute la grotte où repose la sœur de la mère. La peinture se réfère donc à un événement du passé qui obsédait Bedford dans les mois ayant précédé l'exécution de l'œuvre. Plusieurs images sont évoquées: l'ancienne ferme Old Bedford aujourd'hui abandonnée, les personnages de sa mère jeune et son enfant métis, la noyade. Ce drame familial témoigne de la période de la frontière coloniale, très présente dans l'œuvre de Bedford. II n'est pas surprenant que ce frère métis ressurgisse au moment où Bedford, par sa trajectoire, se rapprochait de plus en plus du monde des blancs. Le fait que le personnage principal cité soit un little kardiya («petit blanc») et que l'œuvre soit la seule peinture quasi monochrome de sa carrière n'est peut-être pas anodin.

On retrouve ici une dualité temporelle présente dans de nombreuses œuvres de Bedford avec la présence simultanée de deux traces funéraires distinctes. L'une est la sépulture de la tante de l'artiste qui repose dans une grotte, où elle a réintégré sa forme totémique en tant qu'esprit-enfant. L'autre montre le réservoir où le frère métis de Bedford trouva la mort et d'où il hante toujours les lieux et la mémoire de l'artiste comme juwarri n'ayant pu trouver le repos, porteur de l'histoire tragique du conflit colonial. Dans une même œuvre sont donc ici juxtaposés deux modes distincts de réintégration du passé: I'un mythique (fusion avec les ancêtres totémiques), l'autre historique (le juwarri porteur d'une mémoire historique). Les peintures sont les indices de cette superposition des temps et les supports pour passer d'une mémoire à l'autre.

Cette superposition des temps se retrouve dans le concept kija de gemerre, utilisé pour désigner les traces et les scarifications. II permet de penser un mouvement de l'intérieur vers extérieur, comme les deux faces d'une empreinte dans le sol. On retrouve ce principe chez de nombreux groupes aborigènes. D'après Glowczewski, «les catégories warlpiri de "dessous" et "dessus", plutôt que d'être deux registres de temps passé et présent, apparaissent comme un repérage des deux modes de devenir de la mémoire Jukurrpa [...] : le virtuel et l'actuel » (Glowczewski 1991: 95). Le dreaming est un mode opérateur de la mémoire permettant des permutations entre les registres inconscients/virtuels de la mémoire, d'une part, et les niveaux conscients/actualisés, d'autre part, suivant des sauts temporels et des allersretours entre différentes strates mémorielles. Le travail de la mémoire peut aller du présent vers le passé dans un acte de remémoration mais, par l'intermédiaire des rêves et des morts, le passé revient de lui-même dans le présent.

L'œuvre de Bedford condense ainsi certaines caractéristiques du churinga rappelées en introduction, d'où la comparaison avec une capsule temporelle. Visible dans une faille du bâtiment, elle semble en effet s'adresser au visiteur depuis des temps anciens par des signes difficilement compréhensibles. Mais I'histoire coloniale dont cette œuvre est issue montre que la discontinuité temporelle est moins entre un temps mythique originaire, celui du dreaming, et la perception contemporaine du visiteur, qu'entre un mode d'attachement au territoire à travers une mémoire partagée des lieux et une forme brutale d'occupation de la terre et des corps. Thoowoonggoonarrin permet de saisir simultanément, dans une même image, l'inscription dynamique d'un événement dans un lieu et les mouvements de la propre mémoire de l'artiste. Avec ses sauts à travers des temporalités différentes, la peinture 

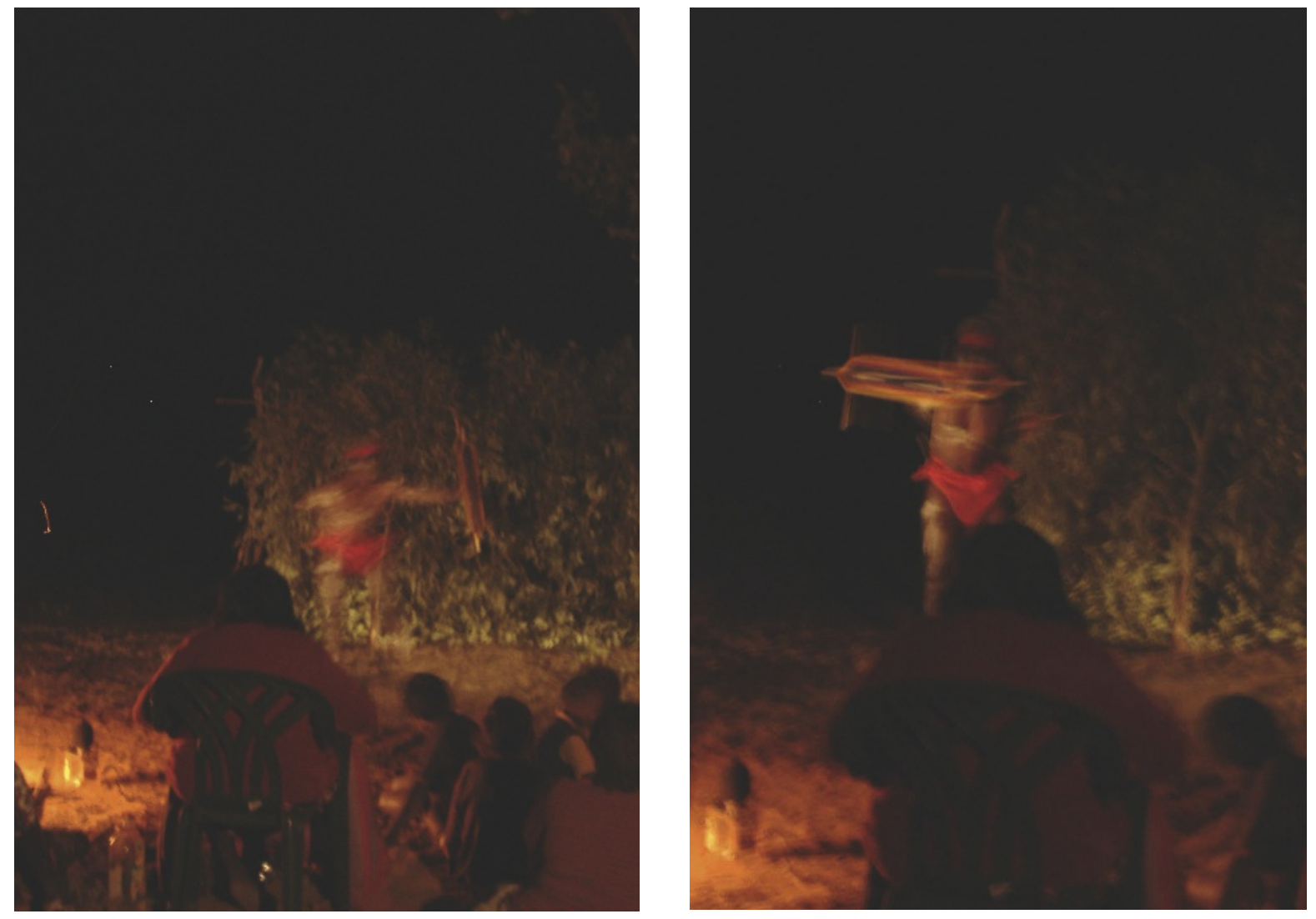

fig. 8

Danse des esprits des morts avec woorrangoo,

joonba du massacre

de Bedford Downs,

communauté de Bow

River, juillet 2007.

Photographies de A

Morvan et K. Rawson. 
de Bedford présente d'indéniables similitudes avec les capsules temporelles mais, contrairement à celles-ci, elle n'est pas fermée sur elle-même, ni dirigée unilatéralement vers un point précis dans un mouvement du présent vers le futur (enterrement de la capsule) ou du présent vers le passé (ouverture de la capsule). Les œuvres de la commande publique condensent ces mouvements dans une superposition dynamique des traces permettant de passer constamment d'un niveau de mémoire à un autre.

On peut alors s'interroger sur la façon dont la commande publique d'art aborigène pour le musée du quai Branly établit des ponts entre les temporalités aborigènes et d'autres formes de temporalités à l'œuvre dans des traditions artistiques issues d'autres sociétés. Si le musée dans son ensemble peut être conçu comme une capsule temporelle adressée aux générations futures en rassemblant des œuvres de l'humanité tout entière, l'analyse d'une œuvre aborigène montre la singularité des pratiques rituelles et artistiques d'un artiste australien contemporain et la diversité parfois conflictuelle des temporalités qui s'y donnent à voir. La commande publique incite à penser les discontinuités temporelles dans l'ensemble des collections tout en replaçant le musée lui-même dans sa propre historicité, une démarche qui permettrait de dépasser l'opposition stérile entre notre temporalité et celle des «autres». 
Bibliographie

\section{Akerman, Kim}

1980 « The Renascence of Aboriginal Law in the Kimberleys", in Ronald M. et Catherine H. Berndt (dir.), Aborigines of the West. Their Past and their Present. Perth, University of Western Australia Press: 234-242.

\section{Armstrong, Claire (éd.)}

2006 Australian Indigenous Art Commission/Commande publique d'Art Aborigène: Musée du quai Branly. Sydney, Art et Australia Pty.

\section{Charlesworth, Max, Dussart, Françoise et Morphy, Howard (éd.)}

2005 Aboriginal Religions in Australia. An Anthology of Recent Writings. Aldershot, Ashgate Publishing.

\section{Coombes, Annie et Phillips, Ruth (éd.)}

2015 Museum Transformations. Chichester, Wiley Blackwell.

\section{De Largy Healy, Jessica}

2010 «Karel Kupka et les maîtres-peintres de la Terre d'Arnhem. La biographie d'une collection d'art aborigène", Gradhiva 12: 198-217.

\section{Derlon, Brigitte et} Jeudy-Ballini, Monique

2001 "Le culte muséal de l'objet sacré ", Gradhiva 30-31 : 202-211.

\section{Di Liberti, Giuseppe}

2013 "Fait/événement Document/monument. La temporalité à l'œuvre ", Images re-vues. Histoire, anthropologie et théorie de l'art 11 [en ligne], disponible sur: https://journals. openedition.org/imagesrevues/3319.

\section{Dolk, Micheal}

2006 "Are we Strangers in this Place? ", in Russell Storer (dir.), Paddy Bedford, catalogue d'exposition. Sydney, Museum of Contemporary Art: 17-49.

\section{Durkheim, Émile}

2008 [1912] Les Formes élémentaires de la vie religieuse. Le Système totémique en Australie. Paris, PUF.

Temporalités encapsulées dans les peintures aborigènes du musée du quai Branly. Par Arnaud Morvan

\section{Eccles, Jeremy}

2004 «Aboriginal Originals Woo French ", Financial Times, 20 décembre.

\section{Elkin, Adolphus P.}

1933 "Totemism in North-Western Australia ", Oceania 3 (3) : 257-296; Oceania 3 (4) : 435-481; Oceania 4 (1): 54-64.

\section{Fabian, Johannes}

1983 Time and the Other. How Anthropology makes its Object. New York, Columbia University Press.

\section{Gell, Alfred}

1992 The Anthropology of Time. Cultural Constructions of Temporal Maps and Images. Oxford, Berg.

\section{Glowczewski, Barbara}

2015 Totemic Becomings. Cosmopolitics of the Dreaming/ Devires totêmicos. Cosmopolítica do sonho. Helsinki/Saõ Paulo, n-1 publications.

1996 « Histoire et ontologie en Australie aborigène ", L'Homme 137: 211-225.

1991 Du rêve à la loi chez les Aborigènes. Mythes, rites et organisation sociale en Australie. Paris, PUF.

\section{Harrison, Robert P.}

2003 The Dominion of the Dead. Chicago, The University of Chicago Press.

\section{Jarvis, William E.}

2003 Time Capsules. A Cultural History. Jefferson, McFarland \& Co.

\section{Kaberry, Phyllis}

1939 Aboriginal Woman. Sacred and Profane. Londres, Routledge \& Sons.

\section{Kaehr, Roland et} Hainard, Jacques (dir.)

1985 Temps perdu, temps retrouvé. Voir les choses du passé au présent. Neuchâtel, Musée d'ethnographie: 51-74.

\section{Keen, Ian}

2005 «Stanner on Aboriginal Religion ", in Maxwell Charlesworth, Françoise Dussart et Howard Morphy (éd.), Aboriginal Religions in Australia. An Anthology of Recent Writings. Aldershot, Ashgate Publishing: 61-78.

\section{Kofod, Frances}

2006 "Paddy Bedford's Stories", in Russell Storer (dir.), Paddy Bedford, catalogue d'exposition. Sydney, Museum of Contemporary Art: 72-98.

2002 «Neminuwarlin Performance Group History ", in Frances Kofod et Tony Oliver (dir.), Fire Fire Burning Bright. Kununurra, Jirrawun Aboriginal Art Corporation: 10-13.

\section{Kopytoff, Igor}

1986 «The Cultural Biography of Things. Commoditization as Process ", in Arjun Appadurai (éd.), The Social Life of Things. Commodities in Cultural Perspective. Cambridge, Cambridge University Press.

\section{Langton, Marcia}

2005 «An Aboriginal Ontology of Being and Place. The Performance of Aboriginal Property Relations in the Princess Charlotte Bay Area of Eastern Cape York Peninsula, Australia". Thèse de doctorat, Sydney, Macquarie University.

Langton, Marcia, Oliver, Tony et Kofod, Frances

2002 Blood on the Spinifex, catalogue d'exposition. Melbourne, The lan Potter Museum of Art/The University of Melbourne.

\section{Lévi-Strauss, Claude}

2008 [1962] La Pensée sauvage, in CEuvres. Paris, Gallimard.

\section{L’Estoile, Benoît (de)}

2007 Le Goût des autres. De l'Exposition coloniale aux arts premiers. Paris, Flammarion.

\section{Macha, Mary et McLeod,} Don

2003 "Introduction ", in Rover Thomas, I Want to Paint. Perth, Heytesbury: 47-51.

\section{Manne, Robert (éd.)}

2003 Whitewash. On Keith Windschuttle's Fabrication of Aboriginal History. Melbourne, Black Inc. Agenda.

\section{Mary, André}

2012 "Retour aux choses sacrées: emblèmes, empreintes et fétiches", Archives de sciences sociales des religions 159: 203-223.

\section{McDonald, Heather}

2010 "Universalising the Particular? God and Indigenous Spirit Being in East Kimberley", TAJA 21 (1): 51-70.

\section{McLean, lan (éd.)}

2011 How Aborigines Invented the Idea of Contemporary Art. Writings on Aboriginal Contemporary Art. Institute of Modern Art and Power Publications, Sydney.

\section{Moisseeff, Marika}

1995 Un long chemin semé d'objets cultuels. Le Cycle initiatique aranda. Paris, Éditions de l'EHESS.

\section{Morvan, Arnaud}

2014 «The East Kimberley Painting Movement: Performing Colonial History ", in Australian Aboriginal Anthropology Today: Critical Perspectives from Europe [en ligne], actes de colloque, disponible sur: http://actesbranly.revues.org/579.

2013 «Mémoires vives de l'ancien et du moderne ", in Arnaud Morvan et Paul Matharan (dir.), Mémoires vives. Une histoire de l'art aborigène, catalogue d'exposition. Paris/ Bordeaux, La Martinière/Musée d'Aquitaine: 19-27.

2012 «La trace chantée dans la peinture kija du Kimberley Oriental ", Cahiers de littérature orale 67-68: 95-119.

2010 "Traces en mouvement. Histoire, mémoire et rituel dans l'art kija contemporain du Kimberley Oriental (nord-ouest australien). ” Thèse de doctorat, Paris/Melbourne, EHESS/University of Melbourne, sous la direction de Barbara Glowczewski et Marcia Langton.

2009 " Performing Landscape and Memory. Gija Local and Global Art Circulation ", in Jaynie Anderson (dir.), Crossing Cultures. Conflict, Migration and Convergence, actes du colloque du Comité international d'histoire de l'art (Melbourne, 2008). Carlton, Melbourne University Press: 823-828. 
Myers, Fred R.

2002 Painting Culture. The Making of an Aboriginal Hight Art. Durham, Duke University Press.

1998 "Question de regard: les expositions d'art aborigène australien en France », Terrain 30: 95-111.

1986 Pintupi Country, Pintupi Self. Sentiment, Place, and Politics among Western Desert Aborigines. Washington/Canberra, Smithsonian Institution Press/Australian Institute of Aboriginal Studies.

\section{Peltier, Philippe}

1998 "Peintures aborigènes

de la terre d'Arnhem en Australie ", Revue du Louvre 48 (2) : 65-69.

\section{Perkins, Hetty} et Croft, Brenda

2006 "Un cadeau au monde entier ", in Claire Armstrong (éd.), Australian Indigenous Art Commission/Commande publique d'Art Aborigène: Musée du quai Branly. Sydney, Art et Australia Pty: 18-21.

\section{Préaud, Martin}

2007 «Deux mises en scène interculturelles de l'histoire ", in Barbara Glowczewski et Rosita Henry (dir.), Le Défi indigène. Entre spectacle et politique. Montreuil, Aux Lieux d'être: 103-124.

\section{Price, Sally}

2007 Paris Primitive. Jacques

Chirac's Museum on the Quai Branly. Chicago, University of Chicago Press.

\section{Radcliffe-Brown, Alfred $R$.}

1931 The Social Organisation of Australian Tribes. Melbourne, MacMillan.

\section{Rumsey, Alan}

1994 "The Dreaming, Human Agency and Inscriptive Practice ", Oceania 65 (2) : 116-130.

Ryan, Judith et Akerman, Kim (éd.)

1993 Images of Power. Aboriginal Art of the Kimberley. Melbourne, National Gallery of Victoria.

\section{Spencer, Baldwin} et Gillen, Francis J.

1899 Native Tribes of Central Australia. London, McMillan.

\section{Stanner, William Edward Hanley}

2009 The Dreaming and Other Essays, Robert Manne (éd.). Melbourne, Black Inc.

1989 [1959] On Aboriginal

Religion, Sydney, Sydney

University Press.

\section{Stanton, John E.}

2001 «Sur l'exposition Marc

Couturier. Secrets ", Gradhiva 30-

31: 199-201.

1986 «Innovation and Change in Aboriginal Art of the Kimberley ", Anthropology News 23 (1) : 4-11.

\section{Strehlow, Ted G.}

1947 Aranda Traditions.

Melbourne, Melbourne

University Press.

\section{Swain, Tony}

1993 A Place for Strangers : Towards a History of Australian Aboriginal Being. Cambridge, Cambridge University Press.

\section{Thomas, Nicholas}

1991 Entangled Objects. Exchange, Material Culture and Colonialism in the Pacific. Cambridge, Harvard University Press.

1999 Possessions. Indigenous Art/Colonial Culture. New York Thames \& Hudson.

\section{Thomas, Northcote W.}

1905 «The Religious Ideas of the Arunta", Folk-Lore 16: 428-433.

\section{Thomas, Rover,} Akerman, Kim, Macha, Mary, Christensen, Will et Caruana, Wally

1994 Roads Cross. The Paintings of Rover Thomas, catalogue d'exposition. Canberra, National Gallery of Australia.

\section{Wolfe, Patrick}

1991 «On Being Woken Up: The Dreamtime in Anthropology and in Australian Settler Culture", Comparative Studies in Society and History 33 (2): 197-224. page 50 et ci-contre

Baldwin Spencer Expédition Horn. Simpsons Gap (Arungachirpa ou Urengetyerrpe), Territoire du nord, Australie, vers 1895, plaque de verre (détail). Collection Museum Victoria, Melbourne @ Museums Victoria Collections

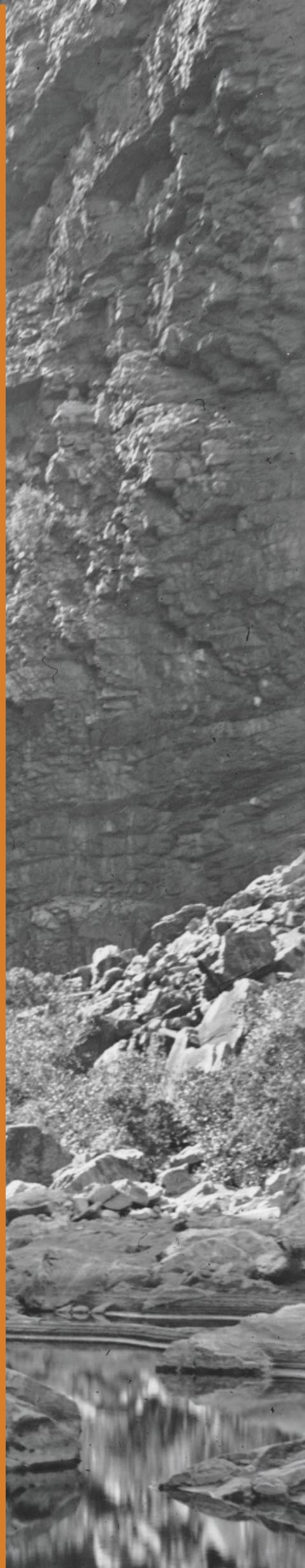

